\title{
Entretien avec Denise Witschard, restauratrice
}

observation, création, collaboration : autour de la restauration du trésor de l'abbaye de Saint-Maurice d'Agaune

\section{Denise Witschard et Natania Girardin}

\section{OpenEdition}

12 Journals

Édition électronique

URL : https://journals.openedition.org/cem/13245

DOI : $10.4000 /$ cem. 13245

ISSN : 1954-3093

Éditeur

Centre d'études médiévales Saint-Germain d'Auxerre

Référence électronique

Denise Witschard et Natania Girardin, «Entretien avec Denise Witschard, restauratrice », Bulletin du centre d'études médiévales d'Auxerre | BUCEMA [En ligne], 17.2 | 2013, mis en ligne le 05 décembre 2013, consulté le 22 septembre 2022. URL : http://journals.openedition.org/cem/13245 ; DOI : https:// doi.org/10.4000/cem. 13245

Ce document a été généré automatiquement le 22 septembre 2022.

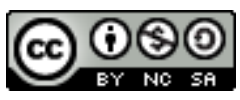

Creative Commons - Attribution - Pas d'Utilisation Commerciale - Partage dans les Mêmes Conditions 4.0 International - CC BY-NC-SA 4.0

https://creativecommons.org/licenses/by-nc-sa/4.0/ 


\title{
Entretien avec Denise Witschard, restauratrice
}

\author{
Observation, création, collaboration : autour de la restauration du trésor \\ de l'abbaye de Saint-Maurice d'Agaune
}

\section{Denise Witschard et Natania Girardin}

Dans le cadre des commémorations du $1500^{\mathrm{e}}$ anniversaire de la fondation de l'abbaye de Saint-Maurice d'Agaune en 2015, le trésor de l'abbaye vit actuellement une refonte complète de ses salles d'exposition, avec, notamment, un agrandissement de la surface ainsi qu'une restauration systématique de ses objets. Denise Witschard, restauratrice, nous ouvre les portes bien gardées de l'abbaye

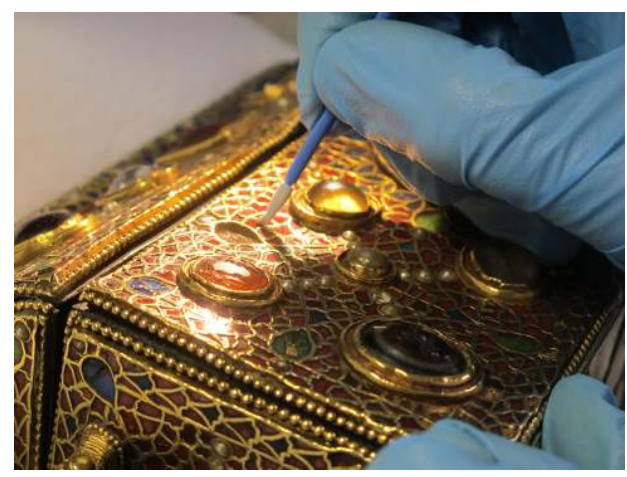
qui protègent une communauté de chanoines réguliers de saint Augustin et leur précieux trésor ${ }^{1}$.

2 Natania Girardin : Pour commencer, pourrais-tu nous parler de tes antécédents, de ta formation professionnelle avant ton engagement à l'abbaye de Saint-Maurice?

Denise Witschard : Je n'ai pas de formation académique car lorsque j'étais étudiante, à la fin des années 1970, il n'existait pas encore en Suisse de formation en conservationrestauration. À l'époque, quelques musées suisses s'étaient regroupés pour offrir une formation commune ${ }^{2}$. J'ai débuté au musée d'Art et d'Histoire de Genève (MAHG), dans l'atelier de restauration fraîchement créé par François Schweizer, spécialisé dans la restauration du métal, où j'ai pu mettre à profit ma formation initiale de bijoutièrejoaillière. Je m'étais également intéressée à la restauration de peinture parce que j'ignorais les autres possibilités qu'il y avait en Suisse, mais l'un des responsables de la Société des restaurateurs suisses m'a dit : «Il faut absolument rester dans votre domaine, on va manquer de gens!» En effet, il avait complètement raison. J'ai eu la chance de pouvoir compléter ma formation en allant faire des stages à l'étranger, notamment 
dans un cours organisé par le Conseil de l'Europe sur la caractérisation des métaux anciens.

4 C'est par la suite que j'ai fait une spécialisation dans la restauration des métaux précieux. Je suis allée me former à la $\mathrm{HEAD}^{3}$, anciennement école des Beaux-arts de Genève, où je connaissais une des professeurs qui recevait toutes les semaines un orfèvre à l'école. J'ai entrepris de faire une formation d'orfèvre, qui est une formation proche, mais différente de celle de bijoutier-joaillier. Cette expérience était fantastique, d'autant plus que j'avais entendu qu'une commission pour la restauration de deux châsses médiévales se mettait sur pied, et je me suis dit qu'il fallait anticiper. Quand la fondation s'est créée, j'ai candidaté ${ }^{4}$, car je voulais avoir l'occasion de travailler dans ce domaine particulier. J'ai été engagée dans le projet, moyennant une spécialisation dans le domaine précis de la restauration des châsses. Pour ce faire, je me suis rendue à Aixla-Chapelle et à Cologne afin de collaborer avec les maîtres orfèvres de ces deux trésors prestigieux. Le projet a duré dix ans, publication comprise ${ }^{5}$. Au sein du MAHG, je travaillais pour le compte de la fondation indépendamment, mais toujours entourée d'une équipe de chercheurs, d'historiens, qui faisaient partie de la commission scientifique mise sur pied dans le cadre de ce grand projet. Au début des années 2000, dans le paysage de la conservation-restauration, ce que nous avons vécu était assez fantastique. C'est une expérience complètement différente de se pencher sur un objet de culte, comme la châsse ${ }^{6}$, que de travailler sur les collections d'un musée.

Cela a donc marqué les prémices de ma collaboration avec l'abbaye de Saint-Maurice.

6 NG : Une fois cette collaboration terminée, comment s'est déroulé ton engagement pour la restauration systématique des objets du trésor de l'abbaye?

7 DW : Une association, Europae thesauri ${ }^{7}$, a contacté l'abbaye afin qu'elle rejoigne ses rangs. Dans ce cadre, Daniel Thurre ${ }^{8}$, l'un des membres de la commission scientifique actuelle, a été approché par Monseigneur Joseph Roduit, abbé de Saint-Maurice, pour entamer une discussion avec l'association. Tout de suite, Daniel Thurre a souhaité que je fasse partie de ces discussions et c'est donc par ce biais que j'ai commencé une nouvelle collaboration avec l'abbaye. Les discussions concernant le $1500^{\mathrm{e}}$ anniversaire de l'abbaye ont commencé à naître, notamment autour de la nécessité de créer un atelier de restauration, en plus d'une commission scientifique pour le trésor. Après de nombreuses tractations - tout ce processus s'est déroulé très lentement -, j'ai eu une assez grande liberté pour composer, en utilisant mon réseau, une commission scientifique avec des personnes qui me paraissaient adéquates ${ }^{9}$, des gens avec qui je travaillerais volontiers. Après trois ans et demi de travail de la commission scientifique, l'atelier a pu voir le jour en janvier 2012.

8 NG: Après une année et demie d'existence, l'atelier a beaucoup de travail actuellement: quels sont les enjeux des restaurations sur les objets du trésor? On a abordé le cas de la châsse des enfants de saint Sigismond, mais pour les autres objets?

9 DW : Concernant les objets à proprement parler, il y a tout d'abord une immense différence entre un objet de musée dont l'histoire est arrêtée et un objet dont l'histoire continue et se crée jour après jour, une différence d'esprit et de conception. En entamant la restauration d'un objet du trésor, tu as entre les mains un objet « vivant». La châsse des enfants de saint Sigismond, avant de venir à Genève dans l'atelier de restauration et d'être touchée par des mains profanes, a été désacralisée et vidée de son 
contenu et des reliques qu'elle contenait ${ }^{10}$. Actuellement, je travaille sur des reliquaires qui conservent leur précieux contenu ${ }^{11}$. L'objet est, de ce fait, tout différent.

Restaurer une châsse est un travail de longue haleine - j'y ai passé trois ans -, car ce n'est pas seulement un objet à restaurer, mais à étudier. On a avancé plus lentement, parce qu'il fallait pouvoir discuter ensemble des différentes étapes. Il y a également eu une exposition de la châsse partiellement restaurée, à moitié démontée, à la fondation Abegg à Riggisberg ${ }^{12}$. L'objectif principal était l'étude de l'objet : il était entendu depuis le départ que nous allions démonter les reliefs puis les déposer parce qu'on était inquiet de l'état du bois sous ces reliefs. Cette dépose a été extrêmement importante pour l'étude de la châsse, car elle nous a permis de documenter les étapes de confection de manière très précise. Nos observations ont été publiées dans l'ouvrage paru après la fin des travaux sur la châsse ${ }^{13}$. Par contre, petite anecdote, nous ne l'avons jamais pesée. On l'a étudiée sous tous ses aspects, mais nous avons oublié de la peser! Quand le nouveau dispositif du trésor sera mis en place, la châsse va pouvoir être pesée : on va lui retirer le socle qui fait partie de la nouvelle vitrine et qui facilite son transport sans que l'on soit obligé de la toucher. À la Saint-Maurice, le 22 septembre, en effet, les châsses sont menées en procession par les chanoines: le contact direct entraîne des dégradations et cela pose de gros problèmes. Malgré cela, la châsse a vécu de nombreuses années. On dit que l'usage des objets est un facteur de dégradation, mais je rappelle chaque fois que c'est aussi un facteur de conservation, parce qu'on vit avec ces objets, on fait attention à eux. Même si de temps en temps, il y a des dégâts, on s'en occupe, on intervient tout de suite, on n'oublie pas.

11 Le fait de travailler au cœur d'une communauté, qui vit avec ces objets et les utilise, permet aussi de se retrouver dans des situations anecdotiques extraordinaires: par exemple, avant la Fête-Dieu, tout à coup, l'après-midi, un chanoine vient avec un ostensoir ${ }^{14}$ dans l'atelier de restauration et me dit : «Il est tout sale, il faut le nettoyer, nous allons l'utiliser demain! ». Je l'ai nettoyé. Ce n'était pas un gros travail, il avait surtout pris la poussière, je l'ai rendu présentable. Ce contact avec la communauté et la liberté qu'ils ont d'aller dans leur trésor prendre un objet à mains nues et de me l'amener, je trouve cela unique.

12 NG : Tu t'occupes d'objets précieux, parfois très anciens, peux-tu nous familiariser avec la façon dont tu procèdes pour entamer la restauration sur un objet? Nous avons sous les yeux, dans ton atelier, le bras-reliquaire de saint Bernard (vers 1180), dont la restauration vient de se terminer.

DW : D'abord, c'est l'observation qui prime. Je me suis aperçue avec l'expérience, que même si on fait une observation très systématique d'un objet, on ne le voit pas tout de suite. On ne le voit que petit à petit, avec le temps. Quand je restaure un objet pendant plusieurs mois, je remarque des détails qui m'avaient échappé à l'origine. Je me rends compte aussi de l'importance d'une bonne campagne photographique préalable, qui permet de s'approprier l'objet, de minimiser l'appréhension que l'on peut ressentir au début du processus de travail; l'observation me permet de surpasser cette peur, d'apprivoiser l'objet.

La seconde étape cruciale est de faire un constat d'état, dans lequel on consigne tous les dégâts, les corrosions, etc. Ce constat permet ensuite d'établir un plan de restauration qui va permettre de mettre à jour les interventions nécessaires. Dans le cas du brasreliquaire, il y avait une ternissure importante de l'argent que j'ai donc consignée. Je savais que je devrais travailler avec des méthodes différentes, qui nécessiteraient des 
outils précis. Ainsi, une méthode d'élimination des couches de corrosions par l'électrochimie a été mise sur pied. On avait déjà travaillé avec cette méthode sur la châsse des enfants de saint Sigismond, mais par trempage. Ici, on savait qu'on aurait besoin d'utiliser cette méthode sans recourir au trempage.

Grâce à la collaboration fructueuse avec Christian Degrigny, de la Haute-École Arc de Neuchâtel, la technique a été mise au point simultanément à la restauration en atelier. J'ai commencé à travailler sur des objets qui pouvaient se restaurer par trempage, qui n'avaient pas d'âme en bois et qui pouvaient être démontés et immergés dans des bains, ce qui était le cas de la statuette équestre de saint Maurice (vers 1577) (fig. 1). Cela nous a pris du temps pour mettre au point cette méthode particulière d'électrochimie : il s'agit d'un pinceau électrolytique qui permet d'intervenir directement sur l'objet sans le mouiller en entier et de s'occuper de la surface sans dégrader le support. Au départ, c'était un prototype peu pratique, mais avec le travail de l'équipe de la Haute-École Arc, il est devenu très efficace et de plus en plus précis (fig. 2). On savait, notamment dans le cas du bras-reliquaire, qu'on ne serait pas obligé de déposer les reliefs par cette méthode alternative au trempage.

Fig. 1 - Denise Witschard en train de nettoyer la statue de saint Maurice à cheval (๑ Trésor de l'abbaye de Saint-Maurice/Atelier de restauration).

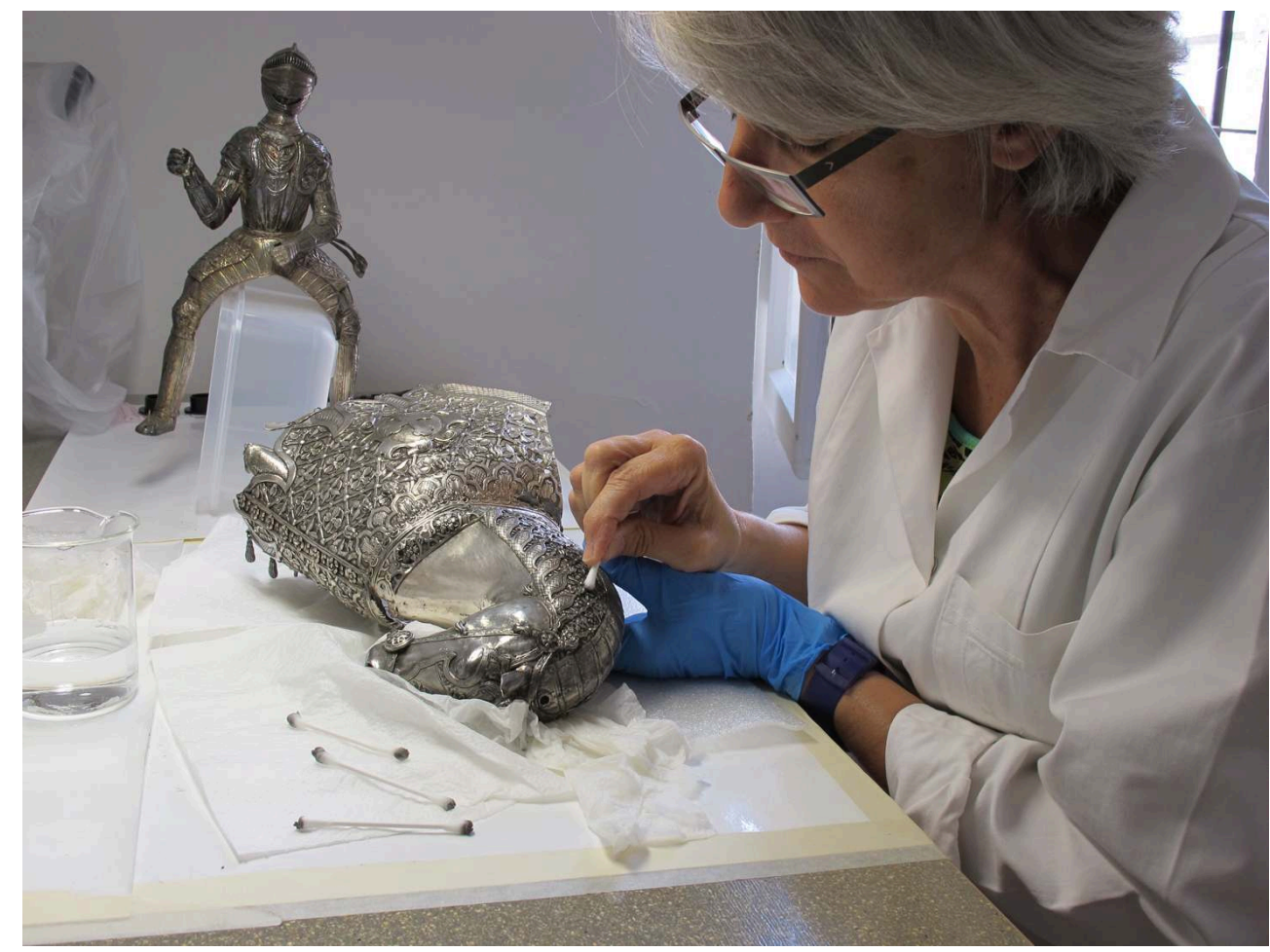


Fig. 2 - Chef reliquaire de saint Candide, XII ${ }^{\mathrm{e}}$ siècle (๔ Trésor de l'abbaye de Saint-Maurice/Atelier de restauration).

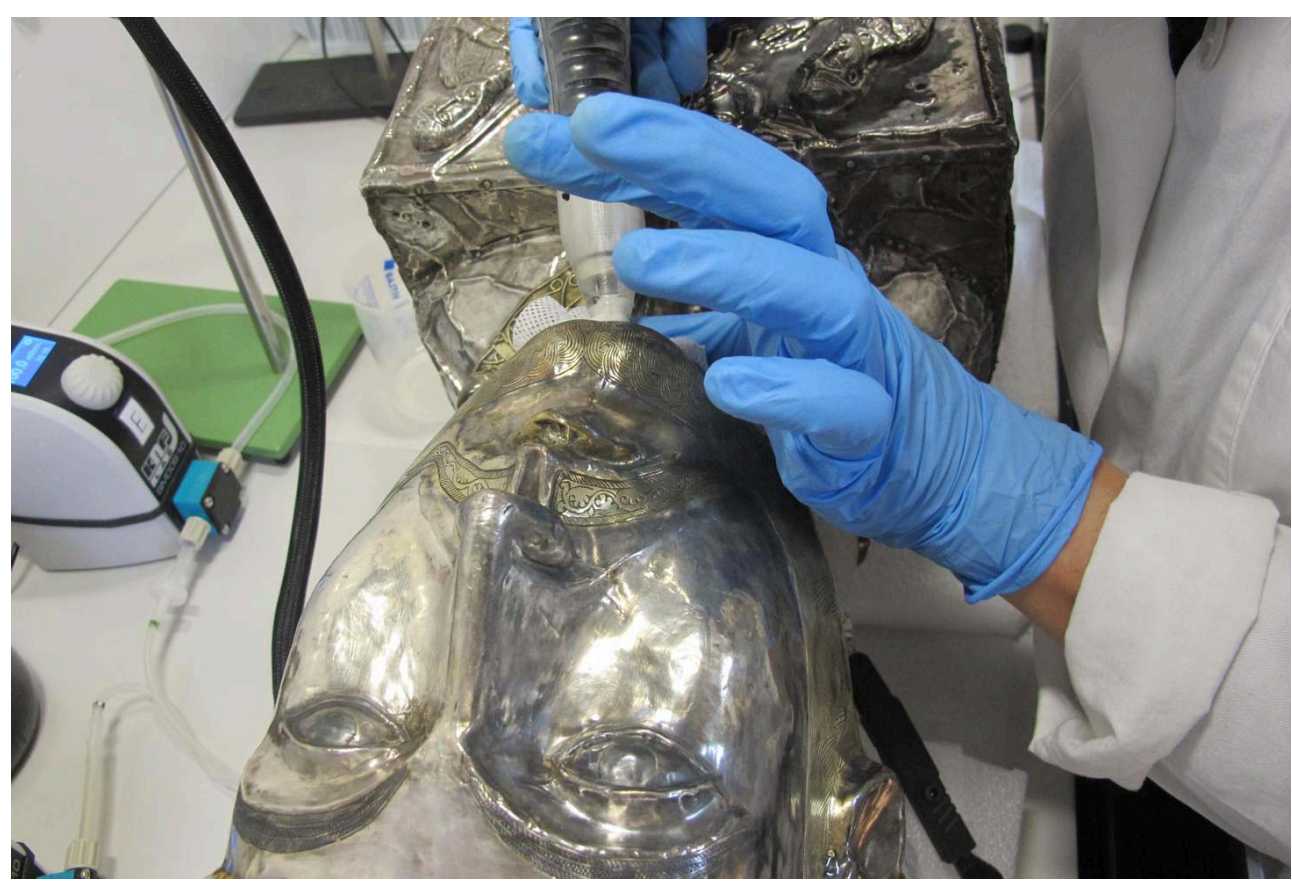

NG : C'est incroyable, parce que cette invention est le résultat direct de la collaboration entre l'atelier de restauration de l'abbaye et la Haute-École Arc.

DW : Oui, c'est ce qui rend notre travail si intéressant, c'est un défi constant! L'autre avantage de ces collaborations, c'est de pouvoir travailler en toute sécurité avec un appareillage très sophistiqué, ce qu'un atelier de restauration ne peut pas obtenir dans des circonstances habituelles.

Dans le cas particulier du bras-reliquaire, nous avons fait des sondages avec le pinceau pour savoir à quel potentiel on allait devoir traiter la surface. Cette phase nous a permis de commencer doucement, sans prendre de risque, puisque la technique n'est ni invasive, ni dangereuse. Dès lors, nous savions comment allaient se dérouler les opérations. Ensuite, une fois les précautions nécessaires prises, ce n'est plus qu'une question de patience, car le travail est relativement long.

Les autres parties du bras-reliquaire - les plaques de cuivre et les émaux par exemple ont été nettoyées mécaniquement avec des bâtonnets imbibés d'une solution d'éthanol et d'eau, méthode simple et longue également, sous loupe binoculaire. Le pinceau ne permet pas d'accéder partout : il est inutilisable par exemple sur le nielle, qui est un oxyde d'argent qui se dégrade par électrolyse.

NG : Comment s'est concrétisée cette collaboration essentielle?

DW : J'ai demandé à Christian Degrigny d'intégrer la commission scientifique pour le trésor, parce qu'il était spécialiste de l'électrochimie et que je savais que nous ne pouvions nous passer de cette méthode. Il a proposé de lancer ce projet de collaboration avec la Haute-École Arc, où il enseigne, pour mettre au point cette technique particulière d'électrochimie. Nous avons également fait appel à un spécialiste hollandais, qui a commencé à mettre au point un pinceau, mais qui n'était pas idéal. Il est venu présenter sa méthode pour que les étudiants, qui allaient travailler sur ce pinceau, imaginent des solutions pour améliorer cet outil. Il est donc très 
important d'avoir une équipe de base intéressante. Cette commission scientifique a un rôle essentiel et les relations sont cruciales. Au final, il y a un atelier de restauration avec un restaurateur, mais qui collabore avec beaucoup de spécialistes de l'extérieur. aux chanoines de l'abbaye les résultats. Cela a permis de rendre notre travail plus concret à leurs yeux. Les résultats des études ont parfois donné aux objets des lettres de noblesse! Pour le coffret de Teuderic, par exemple, les plaquettes qui recouvrent le reliquaire étaient considérées comme de la verroterie alors qu'il s'agit de grenats (fig. 3).

Fig. 3 - Coffret de Teudéric, vII siècle (@ Trésor de l'abbaye de Saint-Maurice/Atelier de restauration).

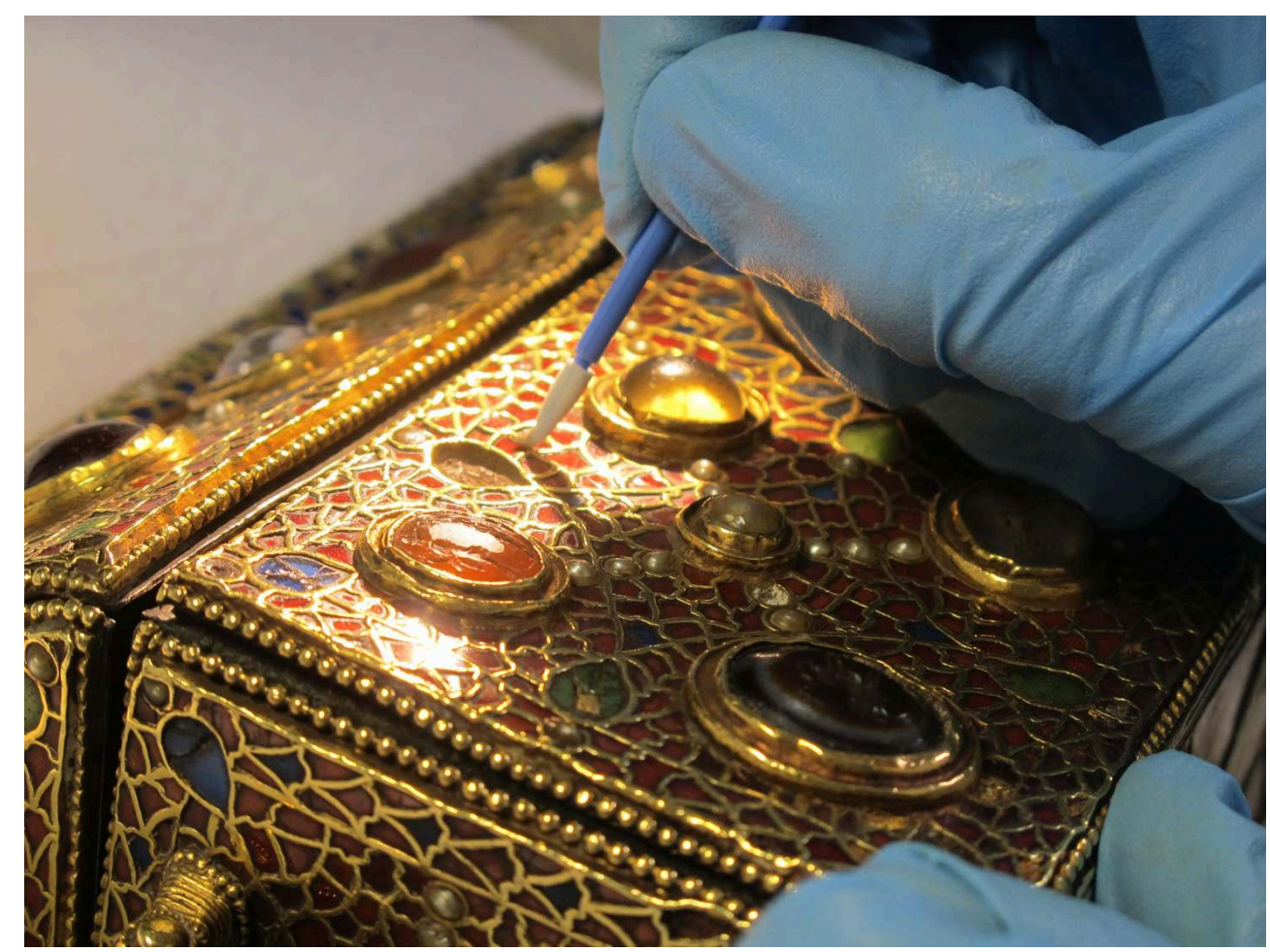

Ces apports, ces découvertes sont cruciaux pour la communauté, mais également pour les historiens d'art, notamment Pierre-Alain Mariaux de l'université de Neuchâtel, 
membre de la commission scientifique, avec lequel nous collaborons étroitement. Tous ces travaux de recherche sur les matériaux constitutifs des objets permettent de mieux comprendre et de mieux situer les objets dans l'histoire.

Notre collaboration est également très précieuse pour moi lorsque je travaille directement sur les objets. Par exemple, je me suis rendu compte, après avoir eu entre les mains plusieurs pièces du trésor, que des soudures à l'étain se retrouvaient sur la majorité d'entre eux. Il y a une telle régularité dans la réparation des objets que cela m'a inévitablement orientée vers les historiens d'art afin de savoir s'il existait des mentions dans les archives d'éventuelles campagnes de restauration. Jusqu'à aujourd'hui, aucune mention n'a été trouvée, mais le travail n'est pas fini. Même si l'on ne trouve aucune mention écrite, l'exploitation de ce que l'on a vu reste essentielle. Cette expérience me permet maintenant de considérer les objets comme constitutif d'un ensemble et traité comme tel par le passé.

NG : Tu penses à une campagne de restauration unique?

DW : Pas forcément, mais en tout cas, au tout début du XIX siècle, quand il n'y avait pas encore le souci de non-intervention sur les objets dans le domaine de la conservationrestauration, on ne disposait pas non plus de colles pour fixer le métal - elles sont apparues dans les années 1950 -, donc une des manières employées pour consolider le métal était la soudure à l'étain. Sur l'argent, c'est un procédé catastrophique, car l'étain dégrade le métal et ne permet pas de retour en arrière. Mais cela fait partie de l'histoire de l'objet et c'est important de le respecter : sans les soudures en étain, la statuette équestre de saint Maurice n'existerait probablement plus à cause des graves dégâts qu'elle a subis. Sans doute, dans des dizaines d'années, on regardera aussi notre travail avec un œil critique, en se disant qu'on aurait pu améliorer certains points. Mais les objets seront toujours là, enfin nous l'espérons, et c'est cela le plus important.

NG : Vous avez déjà bien avancé dans les restaurations des objets. Que reste-t-il à faire avant les grands changements de 2014 avec la réorganisation du trésor et des salles d'exposition?

DW : Notre objectif principal, avant la fin de l'année 2013, était de restaurer tous les objets destinés à la grande exposition Le trésor de Saint-Maurice au musée du Louvre ${ }^{16}$, pendant la dernière période des travaux sur le nouveau trésor. Il fallait absolument que les objets soient en état pour voyager et être exposés. Les conditions de conservation dans l'ancien trésor n'avaient pas permis d'empêcher une légère dégradation des objets, mais on a été satisfait de voir, après le constat général, que seuls quelques objets étaient dans un état préoccupant. À la fin de l'année 2013, les objets pour le Louvre seront terminés, ce qui laissera un peu de temps pour restaurer ceux qui restent. C'est une bonne chose, même dans la nouvelle organisation du trésor, que des objets soient encore à restaurer, afin de montrer qu'il s'agit d'un travail de longue haleine. Il reste beaucoup à faire, dont la restauration des deux autres châsses : celle de saint Maurice et de l'abbé Nantelme ${ }^{17}$. $\mathrm{Si}$, dès le départ, nous savions qu'un apport financier exceptionnel serait nécessaire, la qualité de la restauration des premiers objets sera un atout essentiel pour une levée de fonds supplémentaires.

31 Actuellement, les travaux de fouilles dans l'ancien trésor, qui est entièrement démonté, sont en cours. Les travaux pour la reconstruction et le nouvel espace d'exposition vont pouvoir débuter. 
32 NG : Pour finir, pourrais-tu évoquer ta perception de l'objet en tant que restauratrice, mais également la façon dont tu as saisi la perception des autres acteurs de ce projet autour du trésor de Saint-Maurice?

DW : Quand je pense à l'objet, je pense en premier lieu à sa fonction, à ce qu'il représente. Pour l'artisan, l'objet doit sûrement refléter l'excellence du travail, la beauté des matériaux, sa qualité artistique, mais avec la contrainte toujours présente de représenter ce que le commanditaire lui demande de faire. L'objet d'art considéré par l'historien s'apparente à un vecteur de pensées, au reflet d'un savoir, à une fenêtre ouverte sur les us et coutumes d'une période donnée, à un témoin en somme.

Aux yeux des religieux, les objets sont à la fois tout cela, mais plus encore : les reliques qu'ils contiennent leur confèrent un statut tout à fait particulier, ils deviennent vecteurs non plus d'histoire mais de sainteté. Ainsi pour saisir cette sainteté, l'interaction tactile avec l'objet est essentielle; ils appréhendent l'objet tout à fait différemment des autres. Lorsque les reliques des enfants de saint Sigismond ont été replacées dans la châsse après la restauration, j'ai pu constater à quel point la valeur sacrée de l'objet, son esprit, reprenait sa place légitime aux yeux de la communauté.

Reçu : 30 août 2013. - Accepté : 25 novembre 2013.

\section{NOTES}

1. Entretien mené par Natania Girardin, doctorante à l'Institut d'histoire de l'art et de muséologie, collaboratrice du professeur Pierre-Alain Mariaux dans le projet de réorganisation du trésor de l'abbaye. L'entretien s'est déroulé dans l'atelier de restauration de l'abbaye de Saint-Maurice, le jeudi 22 août 2013.

2. Musées d'art et d'histoire de Genève et de Neuchâtel, musée des Beaux-arts des Grisons, Landesmuseum Zürich.

3. Haute-École d'art et de design de Genève.

4. Fondation pour la restauration de deux chefs-d'œuvre d'orfèvrerie médiévale en Valais, l'autre châsse dont il est question est la Grande châsse de Sion (VS).

5. F. SCHWEIZER et D. WITSCHARD (éd.), La châsse des enfants de saint Sigismond de l'abbaye de SaintMaurice, Paris, 2007.

6. Il s'agit ici de la châsse des enfants de saint Sigismond (XII ${ }^{\mathrm{e}}$-XIII ${ }^{\mathrm{e}}$ siècle).

7. Association internationale des trésors et des musées d'églises en Europe, dont le but est de recenser et de promouvoir la connaissance du patrimoine religieux européen.

8. Historien d'art et conservateur honoraire du trésor de l'abbaye, auteur d'une monographie sur l'atelier roman d'orfèvrerie à l'abbaye de Saint-Maurice d'Agaune.

9. Commission scientifique pour le redéploiement et la restauration du trésor de l'abbaye de Saint-Maurice, à l'occasion du $1500^{\mathrm{e}}$ anniversaire de la fondation de l'abbaye.

10. Après la restauration, les reliques ont été redéposées dans la châsse des enfants de saint Sigismond. 
11. Il est intéressant de noter que les objets du trésor exposés au musée du Louvre ne seront pas désacralisés: ils conserveront leur contenu, comme par exemple la bourse-reliquaire carolingienne.

12. Trésors en péril. Restauration et étude de deux châsses-reliquaire valaisannes du haut Moyen Âge, exposition temporaire du 9 mai au $1^{\mathrm{er}}$ novembre 1999, fondation Abegg, Riggisberg (en collaboration avec le musée d'Art et d'Histoire de Genève).

13. Voir note 5 .

14. Euvre de Marcel Feuillat (1896-1962).

15. Le coffret de Teudéric date du milieu du viI ${ }^{\mathrm{e}}$ siècle; la bourse-reliquaire de la fin du viII $\mathrm{e}_{-}$ début $\mathrm{IX}^{\mathrm{e}}$ siècle ; l'aiguière dite de Charlemagne, du IX ${ }^{\mathrm{e}}$ siècle pour le châssis orfévré et des VII $\mathrm{e}_{-}$ $\mathrm{VIII}^{\mathrm{e}}$ siècles pour les émaux; enfin le vase dit de saint Martin peut être daté du $\mathrm{I}^{\mathrm{er}}$ siècle av. J.C. pour le vase et des $\mathrm{V}^{\mathrm{e}}-\mathrm{VI}^{\mathrm{e}}$ siècles pour la monture.

16. Entre le 14 mars et le 16 juin 2014.

17. Châsse de saint Maurice, $\mathrm{XII}^{\mathrm{e}}$-XIII ${ }^{\mathrm{e}}$ siècle ; châsse de l'abbé Nantelme, 1225.

INDEX

Mots-clés : trésor, restauration

Index géographique : Suisse/Saint-Maurice d'Agaune 